\title{
A note on the approximate controllability of second-order integro-differential evolution control systems via resolvent operators
}

\author{
Velusamy Vijayakumar ${ }^{1}$, Anurag Shukla ${ }^{2}$, Kottakkaran Sooppy Nisar ${ }^{3 *}$, Wasim Jamshed ${ }^{4}$ and \\ Shahram Rezapour ${ }^{5,6^{*}}$ (1)
}

${ }^{*}$ Correspondence:

n.sooppy@psau.edu.sa;

sh.rezapour@azaruniv.ac.ir;

sh.rezapour@mail.cmuh.org.tw;

rezapourshahram@yahoo.ca

${ }^{3}$ Department of Mathematics,

College of Arts and Sciences, Prince

Sattam bin Abdulaziz University,

Wadi Aldawaser 11991, Saudi Arabia

${ }^{5}$ Department of Mathematics,

Azarbaijan Shahid Madani

University, Tabriz, Iran

Full list of author information is

available at the end of the article

\begin{abstract}
The approximate controllability of second-order integro-differential evolution control systems using resolvent operators is the focus of this work. We analyze approximate controllability outcomes by referring to fractional theories, resolvent operators, semigroup theory, Gronwall's inequality, and Lipschitz condition. The article avoids the use of well-known fixed point theorem approaches. We have also included one example of theoretical consequences that has been validated.
\end{abstract}

MSC: Primary 34K30; 34K40; secondary 47H08; 47H10

Keywords: Approximate controllability; Gronwall's inequality; Resolvent operators; Second-order integro-differential systems

\section{Introduction}

The memory effect of the system must be accounted for in numerous disciplines, such as nuclear reactor dynamics and thermoelasticity. The impact of history is overlooked when differential equations, which involve functions at any specific time and space, are used to model such systems. As a result, an integro-differential system is created by adding an integration term to the differential system to include the memory possessions in these frameworks. Integro-differential systems have been widely employed in viscoelastic mechanics, fluid dynamics, thermoelastic contact, control theory, heat conduction, industrial mathematics, financial mathematics, biological models, and other domains, one can refer to $[1-7]$.

Grimmer started and showed the existence of integro-differential systems using resolvent operators in $[1,8,9]$. For solving integro-differential equations, the resolvent operator via fixed-point technique is very easy and most suitable one [10-37]. We recommend readers to $[1,2,8,9,38-42]$ and the sources referenced therein for more information on resolvent operators and integro-differential systems. Very recently in [4], the author presented the controllability of integro-differential inclusions via resolvent operators by employing the facts connected with resolvent operators and Bohnenblust-Karlin's fixed point

(c) The Author(s) 2021. This article is licensed under a Creative Commons Attribution 4.0 International License, which permits use, sharing, adaptation, distribution and reproduction in any medium or format, as long as you give appropriate credit to the original author(s) and the source, provide a link to the Creative Commons licence, and indicate if changes were made. The images or other third party material in this article are included in the article's Creative Commons licence, unless indicated otherwise in a credit line to the material. If material is not included in the article's Creative Commons licence and your intended use is not permitted by statutory regulation or exceeds the permitted use, you will need to obtain permission directly from the copyright holder. To view a copy of this licence, visit http://creativecommons.org/licenses/by/4.0/. 
approach. Also very recently in [2], the authors proved the existence and controllability results for the integro-differential frameworks by applying resolvent operator theories and various fixed point theorems.

Because it is linked to pole assignment, quadratic optimal control, observer design, and other ideas, controllability is significant in mathematical control theories and technical sectors. In infinite-dimensional systems, the two fundamental principles of controllability that can be distinguished are exact and approximation controllability. This is because there are non-closed linear subspaces in infinite-dimensional spaces. Exact controllability enables the system to be directed to any ultimate state, whereas approximate controllability enables it to be derived to any smaller neighborhood of the ultimate state. Many researchers have published about the controllability debate for fractional and integer order frameworks, see [5, 6, 43-60].

Let us consider the following nonlinear differential evolution equations with control:

$$
\begin{aligned}
z^{\prime \prime}(t)= & A(t) z(t)+\int_{0}^{t} \mathscr{B}(t, s) z(s) d s+B v(t) \\
& +F(t, z(t)), \quad t \in J=[0, c] \\
z(0)= & z_{0}, \quad z^{\prime}(0)=z_{1},
\end{aligned}
$$

where $A(t): D(A(t)) \subseteq X \rightarrow X$ and $\mathscr{B}(t, s): D(\mathscr{B}) \subseteq X \rightarrow X$ are closed linear operators in the Hilbert space $X . v(\cdot) \in L^{2}(J, U)$ is a Hilbert space of admissible control functions corresponding to Hilbert space $U$. Additionally, the linear operator $B: U \rightarrow X$ is bounded and $F: J \times X \rightarrow X$. Let us consider that $D(\mathscr{B})$ is independent of $(t, s)$.

The linear type system for the above nonlinear differential evolution equations with control (1.1) - (1.2) is presented by

$$
\begin{aligned}
z^{\prime \prime}(t) & =A(t) z(t)+\int_{0}^{t} \mathscr{B}(t, s) z(s) d s+B v(t), \quad t \in J=[0, c] \\
z(0) & =z_{0}, \quad z^{\prime}(0)=z_{1} .
\end{aligned}
$$

\section{Motivations and contributions:}

- We study the necessary conditions for the approximate controllability of the proposed system (1.1)-(1.2) by using two different conditions.

- In the first condition, we use $B$ in system (1.1)-(1.2) as an $I$ (identity operator), the sufficient conditions for controllability of integro-differential system are discussed.

- In the second condition, we use $B$ in system (1.1)-(1.2) is any bounded operator, the sufficient conditions for controllability of integro-differential system are discussed.

- Results are obtained with the help of Gronwalls' inequality and Lipschitz condition on nonlinearity.

- It is assumed that the resolvent operator is compact, and consequently the associated linear control system is not exactly controllable but only approximately controllable.

- We show that our result has no analog for the concept of complete controllability. To the best of our knowledge, an approximate controllability result has not been studied in this connection. 
- The research focused on the approximate controllability of the proposed system (1.1)-(1.2) under consideration that has not been addressed in the literature to our knowledge, and it supports the current findings.

- Finally, we give an example of the system which is not completely controllable, but approximately controllable.

The structure of the article will now be presented as follows:

1. Sect. 2 discusses some fundamental theories on resolvent operators as well as control theory results.

2. We demonstrate the approximate controllability of integro-differential systems using $B=I$ in Sect. 3 .

3. We demonstrate the approximate controllability of integro-differential systems using $B \neq I$ in Sect. 4.

4. Sect. 5 gives an example of how the acquired hypotheses can be validated.

\section{Preliminaries}

In this section, we mention a few results, notations, and lemmas needed to establish our main results. We introduce certain notations which will be used throughout the article without any further mention. The remainder of this content is structured as follows: $(X, \|$. $\|)$ is a Banach space and $A(t), \mathscr{B}(t, s)$ for $0 \leq s \leq t$ are closed linear operators determined on $D(A)$ and $D(\mathscr{B})$, respectively. We assume that $D(A)$ is dense in $X$.

The space $D(A)$ provided with the graph norm induced by $A(t)$ is a Banach space. We will assume that all of these norms are equivalent. A simple condition for obtaining this property is that there exists $\lambda \in \rho(A(t))$, the resolvent set of $A(t)$, so that $(\lambda I-A(t))^{-1}$ : $X \rightarrow D(A)$ is a bounded linear operator.

Nowadays, there has been an expanding enthusiasm for examining the second order initial value problem,

$$
\begin{aligned}
z^{\prime \prime}(t) & =A(t) z(t)+f(t), \quad 0 \leq s, t \leq c, \\
z(s) & =z^{0}, \quad z^{\prime}(s)=z^{1},
\end{aligned}
$$

where $A(t): D(A) \subseteq X \rightarrow X, t \in[0, c]$, is a densely defined closed linear operator. Additionally, $f:[0, c] \rightarrow X$ is an appropriate function. For discussion of this kind, we refer the readers to [3, 61-68]. In many of the articles, the authors discussed the existence of (2.1)(2.2) connected with $S(t, s)$ has the form

$$
z^{\prime \prime}(t)=A(t) z(t), \quad 0 \leq t \leq c .
$$

Let us assume that, for every $z \in D(A), t \mapsto A(t) z$ is continuous. Now, we assume $A(\cdot)$ generates $(S(t, s))_{0 \leq s \leq t \leq c}$, which is discussed by Kozak [64], Definition 2.1 (refer also to Henriquez [69], Definition 1.1).

We refer to these works for a careful study of this issue. We only regard here that $S(\cdot) z$ is continuously differentiable for all $z \in X$ with derivative uniformly bounded on bounded intervals, which in particular implies that there exists $M_{1}>0$ such that

$$
\|S(t+h, s)-S(t, s)\| \leq M_{1}|h|
$$


for all $s, t, t+h \in[0, c]$. We now determine the operator $C(t, s)=-\frac{\partial S(t, s)}{\partial s}$. Consider $f$ : $[0, c] \rightarrow X$ is an integrable function. We now define the mild solution $z:[0, c] \rightarrow X$ of system (2.1)-(2.2) is as follows:

$$
z(t)=C(t, s) z^{0}+S(t, s) z^{1}+\int_{0}^{t} S(t, \xi) f(\xi) d \xi
$$

Next we consider the second-order integro-differential system

$$
\begin{aligned}
z^{\prime \prime}(t) & =A(t) z(t)+\int_{s}^{t} \mathscr{B}(t, \xi) z(\xi) d \xi, \quad s \leq t \leq c, \\
z(s) & =0, \quad z^{\prime}(s)=x \in X
\end{aligned}
$$

for $0 \leq s \leq c$. This problem was discussed in [3]. We denote $\Delta=\{(t, s): 0 \leq s \leq t \leq c\}$.

We now introduce some conditions that the operator $B(\cdot)$ presented in [3] fulfills.

(B1) For each $0 \leq s \leq t \leq c, \mathscr{B}(t, s):[D(A)] \rightarrow X$ is a bounded linear operator, additionally for every $z \in D(A), \mathscr{B}(\cdot, \cdot) z$ is continuous and

$$
\|\mathscr{B}(t, s) z\| \leq b\|z\|_{[D(A)]}
$$

for $b>0$ which is independent of $s, t \in \Delta$.

(B2) There exists $L_{\mathscr{B}}>0$ such that

$$
\left\|\mathscr{B}\left(t_{2}, s\right) z-\mathscr{B}\left(t_{1}, s\right) z\right\| \leq L_{\mathscr{B}}\left|t_{2}-t_{1}\right|\|z\|_{[D(A)]}
$$

for all $z \in D(A), 0 \leq s \leq t_{1} \leq t_{2} \leq c$.

(B3) There exists $b_{1}>0$ such that

$$
\left\|\int_{\zeta}^{t} S(t, s) \mathscr{B}(s, \zeta) z d s\right\| \leq b_{1}\|z\|
$$

for all $z \in D(A)$ and $0 \leq \zeta \leq t \leq c$.

Under these conditions, it has been established that there exists $(\mathcal{R}(t, s))_{t \geq s}$ associated with problem (2.3)-(2.4). From now, we are going to consider that such a resolvent operator exists, and we adopt its properties as a definition.

Definition 2.1 ([3]) A family of bounded linear operators $(\mathcal{R}(t, s))_{t \geq s}$ on $X$ is said to be a resolvent operator for system (2.3)-(2.4) if it satisfies:

(a) The map $\mathcal{R}: \Delta \rightarrow \mathcal{L}(X)$ is strongly continuous, $\mathcal{R}(t, \cdot) z$ is continuously differentiable for all $z \in X, \mathcal{R}(s, s)=0,\left.\frac{\partial}{\partial t} \mathcal{R}(t, s)\right|_{t=s}=I$, and $\left.\frac{\partial}{\partial s} \mathcal{R}(t, s)\right|_{s=t}=-I$.

(b) Assume $x \in D$. The function $\mathcal{R}(\cdot, s) x$ is a solution of system (2.3)-(2.4). This means that

$$
\frac{\partial^{2}}{\partial t^{2}} \mathcal{R}(t, s) x=A(t) \mathcal{R}(t, s) x+\int_{s}^{t} \mathscr{B}(t, \xi) \mathcal{R}(\xi, s) x d \xi
$$

for all $0 \leq s \leq t \leq c$. 
It follows from condition (a) that there are constants $M>0$ and $\widetilde{M}>0$ such that

$$
\|\mathcal{R}(t, s)\| \leq M, \quad\left\|\frac{\partial}{\partial s} \mathcal{R}(t, s)\right\| \leq \tilde{M}, \quad(t, s) \in \Delta .
$$

Moreover, the linear operator

$$
G(t, \zeta) x=\int_{\zeta}^{t} \mathscr{B}(t, s) \mathcal{R}(s, \zeta) x d s, \quad x \in D(A), 0 \leq \zeta \leq t \leq c,
$$

can be extended to $X$. Portraying this expansion by the similar notation $G(t, \zeta), G: \Delta \rightarrow$ $\mathcal{L}(X)$ is strongly continuous, and it is verified that

$$
\mathcal{R}(t, \zeta) x=S(t, \zeta)+\int_{\zeta}^{t} S(t, s) G(s, \zeta) x d s \quad \text { for all } x \in X
$$

The resulting property is that $\mathcal{R}(\cdot)$ is uniformly Lipschitz continuous, that is, there exists $L_{\mathcal{R}}>0$ such that

$$
\|\mathcal{R}(t+h, \zeta)-\mathcal{R}(t, \zeta)\| \leq L_{\mathcal{R}}|h| \quad \text { for all } t, t+h, \zeta \in[0, c]
$$

Let $g: J \rightarrow X$ be an integrable function. The nonhomogeneous problem

$$
\begin{aligned}
& z^{\prime \prime}(t)=A(t) z(t)+\int_{0}^{t} \mathscr{B}(t, s) z(s) d s+g(t), \quad t \in J=[0, c], \\
& z(0)=x^{0}, \quad z^{\prime}(0)=x^{1},
\end{aligned}
$$

was discussed in [3]. We now introduce the mild solution for system (2.5)-(2.6).

Definition 2.2 ([3]) Assume $x^{0}, x^{1} \in X$. The function $z:[0, c] \rightarrow X$ given by

$$
z(t)=-\left.\frac{\partial \mathcal{R}(t, s) x^{0}}{\partial s}\right|_{s=0}+\mathcal{R}(t, 0) x^{1}+\int_{0}^{t} \mathcal{R}(t, s) g(s) d s
$$

is said to be the mild solution for system (2.5)-(2.6).

It is clear that $z(\cdot)$ in Definition 2.2 is a continuous function.

Definition 2.3 A continuous function $z:[0, c] \rightarrow X$ is said to be a mild solution for system (1.1)-(1.2) if $z(0)=z_{0}, z^{\prime}(0)=z_{1}$, and

$$
\begin{aligned}
z(t)= & -\left.\frac{\partial \mathcal{R}(t, s) x^{0}}{\partial s}\right|_{s=0}+\mathcal{R}(t, 0) z_{1}+\int_{0}^{t} \mathcal{R}(t, s) B v(s) d s \\
& +\int_{0}^{t} \mathcal{R}(t, s) F(s, z(s)) d s, \quad t \in J,
\end{aligned}
$$

is fulfilled. 
Definition 2.4 The reachable set of system (1.1)-(1.2) given by

$$
K_{c}(F)=\{z(c) \in X: z(t) \text { represents a mild solution of system }(1.1)-(1.2)\} .
$$

In case $F \equiv 0$, system (1.1)-(1.2) reduces to the corresponding linear system. The reachable set in this case is denoted by $K_{c}(0)$.

Definition 2.5 If $\overline{K_{c}(F)}=X$, then the semilinear control system is approximately controllable on $[0, c]$. Here $\overline{K_{c}(F)}$ represents the closure of $K_{c}(F)$. Clearly, if $\overline{K_{c}(0)}=X$, then the linear system is approximately controllable.

Assume that $\Psi=L^{2}(J, X)$. Define the operator $\aleph: \Psi \rightarrow \Psi$ as follows:

$$
[\aleph z](t)=F(t, z(t)) ; \quad 0<t \leq c
$$

\section{Controllability results when $B=I$}

For this discussion, it is shown that the approximate controllability of the linear system reaches from the semilinear system under specific requirements on the nonlinear term. Clearly, $X=U$.

Assume the following linear system

$$
\begin{aligned}
w^{\prime \prime}(t) & =A(t) w(t)+\int_{0}^{t} \mathscr{B}(t, s) w(s) d s+u(t), \quad t \in J=[0, c] \\
z(0) & =z_{0}, \quad z^{\prime}(0)=z_{1},
\end{aligned}
$$

and the semilinear system

$$
\begin{aligned}
& z^{\prime \prime}(t)=A(t) z(t)+\int_{0}^{t} \mathscr{B}(t, s) z(s) d s+v(t)+F(t, z(t)), \quad t \in J, \\
& z(0)=z_{0}, \quad z^{\prime}(0)=z_{1} .
\end{aligned}
$$

For proving the primary task of this section, that is, the approximate controllability of system (3.3)-(3.4), we need to introduce the following hypotheses:

$\left(\mathbf{H}_{\mathbf{1}}\right)$ Linear system (3.1)-(3.2) is approximately controllable.

$\left(\mathbf{H}_{2}\right) F(t, z(t))$ is a nonlinear function which fulfills the Lipschitz condition in $z$, that is,

$$
\left\|F\left(t, z_{1}\right)-F\left(t, z_{2}\right)\right\|_{X} \leq l\left(\left\|z_{1}-z_{2}\right\|_{X}\right), \quad l>0, \text { for all } z_{1}, z_{2} \in X, t \in J
$$

Theorem 3.1 If hypotheses $\left(\mathbf{H}_{\mathbf{1}}\right)-\left(\mathbf{H}_{\mathbf{2}}\right)$ are satisfied, then (3.3)-(3.4) is approximately controllable.

Proof Assume that $w(t)$ is the mild solution of system (3.1)-(3.2), along with the control $u$. Assume the following semilinear system:

$$
\begin{aligned}
z^{\prime \prime}(t)= & A(t) z(t)+\int_{0}^{t} \mathscr{B}(t, s) z(s) d s+F(t, z(t)) \\
& +u(t)-F(t, w(t)), \quad t \in J,
\end{aligned}
$$




$$
z(0)=z_{0}, \quad z^{\prime}(0)=z_{1}
$$

On comparing system (3.3)-(3.4) and system (3.5)-(3.6), we can see the control function $v(t)$ is assumed such that

$$
v(t)=u(t)-F(t, w(t))
$$

The mild solution of system (3.1)-(3.2) is given by

$$
w(t)=-\left.\frac{\partial \mathcal{R}(t, s) x^{0}}{\partial s}\right|_{s=0}+\mathcal{R}(t, 0) z_{1}+\int_{0}^{t} \mathcal{R}(t, s) u(s) d s, \quad t \in J
$$

and the mild solution of system (3.5)-(3.6) is given by

$$
\begin{aligned}
z(t)= & -\left.\frac{\partial \mathcal{R}(t, s) x^{0}}{\partial s}\right|_{s=0}+\mathcal{R}(t, 0) z_{1}+\int_{0}^{t} \mathcal{R}(t, s)[F(s, z(s))+u(s) \\
& -F(s, w(s))] d s, \quad t \in J .
\end{aligned}
$$

From equation (3.7) and equation (3.8), we get

$$
w(t)-z(t)=\int_{0}^{t} \mathcal{R}(t, s)\{F(s, w(s))-F(s, z(s))\} d s .
$$

Applying norm on both sides, we have

$$
\begin{aligned}
\|w(t)-z(t)\|_{X} & \leq \int_{0}^{t}\|\mathcal{R}(t, s)\|\|F(s, w(s))-F(s, z(s))\| d s \\
& \leq M \int_{0}^{t}\|F(s, w(s))-F(s, z(s))\| d s .
\end{aligned}
$$

Using hypothesis $\left(H_{2}\right)$, we get

$$
\|w(s)-z(s)\|_{X} \leq M l \int_{0}^{t}\|w(s)-z(s)\| d s
$$

By referring to Gronwall's inequality, $w(t)=z(t)$ for all $t \in[0, c]$. Thus, the solution $w$ of the linear system along the control $u$ is a solution of the semilinear system $z$ along the control $v$, i.e., $K_{c}(F) \supset K_{c}(0)$. Because $K_{c}(0)$ is dense in $X$ (by employing hypothesis $\left(H_{1}\right)$ ), $K_{c}(F)$ is dense in $X$ too, which concludes the approximate controllability of system (3.3)-(3.4), and this concludes the proof.

\section{Controllability results of semilinear system when $B \neq I$}

Now, the approximate controllability when $B \neq I$ is verified under certain conditions on $A$, $B$, and $F$.

Assume the following linear system

$$
w^{\prime \prime}(t)=A(t) w(t)+\int_{0}^{t} \mathscr{B}(t, s) w(s) d s+B u(t), \quad t \in J,
$$




$$
z(0)=z_{0}, \quad z^{\prime}(0)=z_{1},
$$

and the semilinear system

$$
\begin{aligned}
& z^{\prime \prime}(t)=A(t) z(t)+\int_{0}^{t} \mathscr{B}(t, s) z(s) d s+B v(t)+F(t, z(t)), \quad t \in J, \\
& z(0)=z_{0}, \quad z^{\prime}(0)=z_{1} .
\end{aligned}
$$

For proving the primary task of this section, that is, the approximate controllability of system (4.3)-(4.4), we have to introduce the following hypotheses:

$\left(\mathbf{H}_{3}\right)$ Linear system (4.1)-(4.2) is approximately controllable.

$\left(\mathbf{H}_{4}\right)$ Range of the operator $\aleph$ is a subset of the closure of range of $B$, i.e.,

$$
\operatorname{Range}(\aleph) \subseteq \overline{\operatorname{Range}(B)}
$$

Theorem 4.1 If hypotheses $\left(\mathbf{H}_{2}\right)-\left(\mathbf{H}_{\mathbf{4}}\right)$ are fulfilled, then system (1.1)-(1.2) is approximately controllable.

Proof The mild solution of system (4.1)-(4.2) corresponding to the control $u$ is given by

$$
w(t)=-\left.\frac{\partial \mathcal{R}(t, s) x^{0}}{\partial s}\right|_{s=0}+\mathcal{R}(t, 0) z_{1}+\int_{0}^{t} \mathcal{R}(t, s) B u(s) d s, \quad t \in J .
$$

Assume the following semilinear system:

$$
\begin{aligned}
z^{\prime \prime}(t)= & A(t) z(t)+\int_{0}^{t} \mathscr{B}(t, s) z(s) d s+F(t, z(t))+B u(t) \\
& -F(t, w(t)), \quad t \in J, \\
z(0)= & z_{0}, \quad z^{\prime}(0)=z_{1} .
\end{aligned}
$$

Since $\aleph z \in \overline{\operatorname{Range}(B)}$, for given $\epsilon>0$, there exists a control function $v \in L^{2}(J, U)$ such that

$$
\|\aleph z-B v\|_{X} \leq \epsilon
$$

Now, assume that $z(t)$ is the mild solution of system (1.1)-(1.2) corresponding to $(u-v)$ given by

$$
z(t)=-\left.\frac{\partial \mathcal{R}(t, s) x^{0}}{\partial s}\right|_{s=0}+\mathcal{R}(t, 0) z_{1}+\int_{0}^{t} \mathcal{R}(t, s)\{B(u-v)+[\aleph z]\}(s) d s, \quad t \in J
$$

From equation (4.5) and equation (4.7), we have

$$
\begin{aligned}
w(t)-z(t) & =\int_{0}^{t} \mathcal{R}(t, s)[B v-z](s) d s \\
& =\int_{0}^{t} \mathcal{R}(t, s)[B v-\aleph w](s) d s+\int_{0}^{t} \mathcal{R}(t, s)[\aleph w-\aleph z](s) d s .
\end{aligned}
$$


Taking norm on both sides and using (4.6), we get

$$
\begin{aligned}
\|w(t)-z(t)\|_{X}= & \int_{0}^{t}\|\mathcal{R}(t, s)\|\|B v(s)-\aleph w(s)\| d s \\
& +\int_{0}^{t}\|\mathcal{R}(t, s)\|\|\aleph w(s)-\aleph z(s)\|_{X} d s \\
\leq & M\left(\int_{0}^{t} d s\right)^{1 / 2}\left(\int_{0}^{t}\|B v(s)-\aleph w(s)\|^{2} d s\right)^{1 / 2} \\
& +M \int_{0}^{t}\|\aleph w(s)-\aleph z(s)\|_{X} \\
\leq & M \sqrt{t}\left(\|\aleph w-B v\|_{L^{2}([0, c] ; X)}\right)+M \int_{0}^{t}\|[\aleph w](s)-[\aleph z](s)\|_{X} d s \\
\leq & M \sqrt{c} \epsilon+M l \int_{0}^{t}\|w(s)-z(s)\|_{X} d s .
\end{aligned}
$$

By referring to Gronwall's inequality, we have

$$
\|w(t)-z(t)\|_{X} \leq M \sqrt{c} \epsilon \exp (M l c)
$$

Since the right-hand side of the above inequality depends on $\epsilon>0$ and $\epsilon$ is arbitrary, it is clear that $\|w(t)-z(t)\|_{X}$ becomes arbitrarily small by selecting a suitable control function $v$. Clearly, the reachable set of system (1.1)-(1.2) is dense in the reachable set of system (4.1)(4.2), which is dense in $X$ due to hypothesis $\left(H_{3}\right)$. Hence, the approximate controllability of system (4.1)-(4.2) implies that of the semilinear control system (1.1)-(1.2).

\section{Example}

Assume that the integro-differential system with control has the form

$$
\begin{aligned}
& \frac{\partial^{2}}{\partial t^{2}} \chi(t, y)=\frac{\partial^{2}}{\partial x^{2}} \chi(t, y)+a(t) \chi(t, y)+\int_{0}^{t} b(t-s) \frac{\partial^{2} \chi(s, y)}{\partial x^{2}} d s \\
& +\mu(t, y)+\frac{\chi^{2}(t, y)}{(1+t)\left(1+t^{2}\right)}, \quad 0 \leq t \leq c, 0 \leq y \leq \pi, \\
& \chi(t, 0)=\chi(t, \pi)=0, \\
& \chi(0, y)=\chi_{0}(y), \quad \frac{\partial}{\partial t} \chi(0, y)=\chi_{1}(y) \quad 0 \leq y \leq \pi,
\end{aligned}
$$

where $a, b:[0, c] \rightarrow \mathbb{R}, c>0$ are continuous functions, $\chi_{0}(y), \chi_{1}(y) \in X=L^{2}([0, \pi])$ and the function $\mu: I \times[0, \pi] \rightarrow[0, \pi]$ is continuous.

We denote by $A_{0}$ the operator given by $A_{0} z(\xi)=z^{\prime \prime}(x)$ with domain

$$
D(A)=\left\{z \in H^{2}([0, \pi]): z(0)=z(\pi)=0\right\} .
$$

Then $A_{0}$ is the infinitesimal generator of a cosine function of operators $\left(C_{0}(t)\right)_{t \in \mathbb{R}}$ on $H$ associated with sine function $\left(S_{0}(t)\right)_{t \in \mathbb{R}}$. Additionally, $A_{0}$ has discrete spectrum which consists of eigenvalues $-n^{2}$ for $n \in \mathbb{N}$, with corresponding eigenvectors

$$
w_{n}(x)=\frac{1}{\sqrt{2 \pi}} e^{i n x}, \quad n \in \mathbb{N} .
$$


The set $\left\{w_{n}: n \in \mathbb{N}\right\}$ is an orthonormal basis of $H$. Applying this idea, we can write

$$
A_{0} z=\sum_{n=1}^{\infty}-n^{2}\left\langle z, w_{n}\right\rangle w_{n}
$$

for $z \in D\left(A_{0}\right),\left(C_{0}(t)\right)_{t \in \mathbb{R}}$ is given by

$$
C_{0}(t) z=\sum_{n=1}^{\infty} \cos (n t)\left\langle z, w_{n}\right\rangle w_{n}, \quad t \in \mathbb{R}
$$

and the sine function is given by

$$
S_{0}(t) z=\sum_{n=1}^{\infty} \frac{\sin (n t)}{n}\left\langle z, w_{n}\right\rangle w_{n}, \quad t \in \mathbb{R}
$$

It is immediate from these representations that $\left\|C_{0}(t)\right\| \leq 1$ and that $S_{0}(t)$ is compact for all $t \in \mathbb{R}$.

We define $A(t) z=A_{0} z+a(t) z$ on $D(A)$. Clearly, $A(t)$ is a closed linear operator. Therefore, $A(t)$ generates $(S(t, s))_{0 \leq s \leq t \leq c}$ such that $S(t, s)$ is compact for all $0 \leq s \leq t \leq c$ ([3]).

We complete the terminology by defining $B(t, s)=b(t-s) A_{0}$ for $0 \leq s \leq t \leq c$ on $D(A)$.

We now assume the function $F: J \times X$ by

$$
\begin{aligned}
& F(t, x)=\frac{\chi^{2}(t, x)}{(1+t)\left(1+t^{2}\right)}, \\
& B v(t, \xi)=\mu(t, \xi) .
\end{aligned}
$$

Let us consider that the above functions meet the hypotheses condition with $B=I$ as shown above. Since all the requirements are fulfilled, then system (5.1)-(5.3) is approximately controllable.

\section{Conclusion}

We primarily focused on the approximate controllability of nonlinear resolvent integrodifferential evolution control systems. We analyzed approximate controllability outcomes for the considered systems by referring to resolvent operators, semigroup theory, Gronwall's inequality, and Lipschitz condition. The article avoids the use of well-known fixed point theorem approaches. The discussion on the approximate controllability of nonlinear resolvent stochastic integro-differential evolution equations with impulses will be our future work.

\section{Acknowledgements}

The fifth author was supported by Azarbaijan Shahid Madani University. The authors express their gratitude to dear unknown referees for their helpful suggestions which improved the final version of this paper.

\section{Funding}

Not applicable. 


\section{Declarations}

\section{Ethics approval and consent to participate}

Not applicable.

\section{Consent for publication}

Not applicable.

\section{Competing interests}

The authors declare that they have no competing interests.

\section{Authors' contributions}

The authors declare that the study was realized in collaboration with equal responsibility. All authors read and approved the final manuscript.

\section{Author details}

'Department of Mathematics, School of Advanced Sciences, Vellore Institute of Technology, Vellore 632 014, Tamil Nadu, India. ${ }^{2}$ Department of Applied Sciences, Rajkiya Engineering College, Kannauj, India. ${ }^{3}$ Department of Mathematics, College of Arts and Sciences, Prince Sattam bin Abdulaziz University, Wadi Aldawaser 11991, Saudi Arabia. ${ }^{4}$ Department of Mathematics, Capital University of Science and Technology (CUST), Islamabad, 44000, Pakistan. ${ }^{5}$ Department of Mathematics, Azarbaijan Shahid Madani University, Tabriz, Iran. ${ }^{6}$ Department of Medical Research, China Medical University Hospital, China Medical University, Taichung, Taiwan.

\section{Publisher's Note}

Springer Nature remains neutral with regard to jurisdictional claims in published maps and institutional affiliations.

Received: 7 August 2021 Accepted: 19 October 2021 Published online: 06 November 2021

\section{References}

1. Desch, W., Grimmer, R., Schappacher, W.: Some considerations for linear integro-differential equations. J. Math. Anal. Appl. 104(1), 219-234 (1984). https://doi.org/10.1016/0022-247X(84)90044-1

2. Murugesu, R., Vijayakumar, V., dos Santos, J.P.C.: Existence of mild solutions for nonlocal Cauchy problem for fractional neutral integro-differential equation with unbounded delay. Commun. Math. Anal. 14(1), 59-71 (2013)

3. Henriquez, H.R., Pozo, J.C.: Existence of solutions of abstract non-autonomous second order integro-differential equations. Bound. Value Probl. 2016, 168 (2016). https://doi.org/10.1186/s13661-016-0675-7

4. Vijayakumar, V:: Approximate controllability results for analytic resolvent integro-differential inclusions in Hilbert spaces. Int. J. Control 91(1), 204-214 (2018). https://doi.org/10.1080/00207179.2016.1276633

5. Vijayakumar, V., Selvakumar, A., Murugesu, R.: Controllability for a class of fractional neutral integro-differential equations with unbounded delay. Appl. Math. Comput. 232, 303-312 (2014). https://doi.org/10.1016/j.amc.2014.01.029

6. Vijayakumar, V., Ravichandran, C., Murugesu, R., Trujillo, J.J.: Controllability results for a class of fractional semilinear integro-differential inclusions via resolvent operators. Appl. Math. Comput. 247(4), 152-161 (2014). https://doi.org/10.1016/j.amc.2014.08.080

7. Sivasankaran, S., Arjunan, M.M., Vijayakumar, V.: Existence of global solutions for second order impulsive abstract partial differential equations. Nonlinear Anal., Theory Methods Appl. 74(17), 6747-6757 (2011). https://doi.org/10.1016/j.na.2011.06.054

8. Grimmer, R:: Resolvent operators for integral equations in a Banach space. Trans. Am. Math. Soc. 273(1), 333-349 (1982). https://doi.org/10.2307/1999209

9. Grimmer, R., Pritchard, A.J.: Analytic resolvent operators for integral equations in a Banach space. J. Differ. Equ. 50(2), 234-259 (1983). https://doi.org/10.1016/0022-0396(83)90076-1

10. Abdeljawad, T., Agarwal, R.P., Karapinar, E., Kumari, P.S.: Solutions of the nonlinear integral equation and fractional differential equation using the technique of a fixed point with a numerical experiment in extended b-metric space. Symmetry 11(5), 686 (2019). https://doi.org/10.3390/sym11050686

11. Adiguzel, R.S., Aksoy, U., Karapinar, E., Erhan, I.M.: On the solution of a boundary value problem associated with a fractional differential equation. Math. Methods Appl. Sci. (2020). https://doi.org/10.1002/mma.6652

12. Adiguzel, R.S., Aksoy, U., Karapinar, E., Erhan, I.M.: Uniqueness of solution for higher-order nonlinear fractional differential equations with multi-point and integral boundary conditions. Rev. R. Acad. Cienc. Exactas Fís. Nat., Ser. A Mat. 2021, 155 (2021). https://doi.org/10.1007/s13398-021-01095-3

13. Adiguzel, R.S., Aksoy, U., Karapinar, E., Erhan, I.M.: On the solutions of fractional differential equations via Geraghty type hybrid contractions. Appl. Comput. Math. 20(2), 313-333 (2021)

14. Karapinar, E., Fulga, A.: An admissible hybrid contraction with an Ulam type stability. Demonstr. Math. 52, 428-436 (2019). https://doi.org/10.1515/dema-2019-0037

15. Alqahtani, B., Fulga, A., Karapinar, E.: Fixed point results on $\delta$-symmetric quasi-metric space via simulation function with an application to Ulam stability. Mathematics 6(10), 208 (2018). https://doi.org/10.3390/math6100208

16. Brzdek, J., Karapinar, E., Petrsel, A.: A fixed point theorem and the Ulam stability in generalized dq-metric spaces. J. Math. Anal. Appl. 467, 501-520 (2018). https://doi.org/10.1016/j.jmaa.2018.07.022

17. Hassan, A.M., Karapinar, E., Alsulami, H.H.: Ulam-Hyers stability for MKC mappings via fixed point theory. J. Funct. Spaces 2016, Article ID 9623597 (2016). https://doi.org/10.1155/2016/9623597

18. Rezapour, S., Azzaoui, B., Tellab, B., Etemad, S., Masiha, H.P.: An analysis on the positivesSolutions for a fractional configuration of the Caputo multiterm semilinear differential equation. J. Funct. Spaces 2021, Article ID 6022941 (2021). https://doi.org/10.1155/2021/6022941 
19. Sabetghadam, F., Masiha, H.P.: Fixed-point results for multi-valued operators in quasi-ordered metric spaces. Appl. Math. Lett. 25(11), 1856-1861 (2012). https://doi.org/10.1016/j.aml.2012.02.046

20. Masiha, H.P., Sabetghadam, F., Shahzad, N.: Fixed point theorems in partial metric spaces with an application. Filomat 27(4), 617-624 (2013)

21. Sabetghadam, F., Masiha, H.P.: Common fixed points for generalized $\varphi$-pair mappings on cone metric spaces. Fixed Point Theory Appl. 2010, Article ID 718340 (2010). https://doi.org/10.1155/2010/718340

22. Baleanu, D., Jajarmi, A., Mohammadi, H., Rezapour, S.: A new study on the mathematical modelling of human liver with Caputo-Fabrizio fractional derivative. Chaos Solitons Fractals 134, 109705 (2020). https://doi.org/10.1016/j.chaos.2020.109705

23. Baleanu, D., Mohammadi, H., Rezapour, S.: Analysis of the model of HIV-1 infection of CD4 ${ }^{+}$T-cell with a new approach of fractional derivative. Adv. Differ. Equ. 2020, 71 (2020). https://doi.org/10.1186/s13662-020-02544-w

24. Dineshkumar, C., Udhayakumar, R., Vijayakumar, V., Nisar, K.S.: A discussion on the approximate controllability of Hilfer fractional neutral stochastic integro-differential systems. Chaos Solitons Fractals 142, 110472 (2021). https://doi.org/10.1016/j.chaos.2020.110472

25. Dineshkumar, C., Udhayakumar, R., Vijayakumar, V., Nisar, K.S., Shukla, A.: A note on the approximate controllability of Sobolev type fractional stochastic integro-differential delay inclusions with order $1<r<2$. Math. Comput. Simul. 190 1003-1026 (2021). https://doi.org/10.1016/j.matcom.2021.06.026

26. Kim, I.S.: Semilinear problems involving nonlinear operators of monotone type. Results Nonlinear Anal. 2(1), 25-35 (2019)

27. Lazreg, J.E., Abbas, S., Benchohra, M., Karapinar, E.: Impulsive Caputo-Fabrizio fractional differential equations in b-metric spaces. Open Math. 19(1), 363-372 (2021). https://doi.org/10.1515/math-2021-0040

28. Rezapour, S., Mohammadi, H., Jajarmi, A.: A new mathematical model for Zika virus transmission. Adv. Differ. Equ. 2020, 589 (2020). https://doi.org/10.1186/s13662-020-03044-7

29. Mohammadi, H., Kumar, S., Rezapour, S., Etemad, S.: A theoretical study of the Caputo-Fabrizio fractional modeling for hearing loss due to Mumps virus with optimal control. Chaos Solitons Fractals 144, 110668 (2021). https://doi.org/10.1016/j.chaos.2021.110668

30. Rezapour, S., Imran, A., Hussain, A., Martinez, F., Etemad, S., Kaabar, M.K.A.: Condensing functions and approximate endpoint criterion for the existence analysis of quantum integro-difference FBVPs. Symmetry 13(3), 469 (2021). https://doi.org/10.3390/sym13030469

31. Matar, M.M., Abbas, M.I., Alzabut, J., Kaabar, M.K.A., Etemad, S., Rezapour, S.: Investigation of the p-Laplacian nonperiodic nonlinear boundary value problem via generalized Caputo fractional derivatives. Adv. Differ. Equ. 2021 68 (2021). https://doi.org/10.1186/s13662-021-03228-9

32. Thabet, S.T.M., Etemad, S., Rezapour, S.: On a coupled Caputo conformable system of pantograph problems. Turk. J. Math. 45(1), 496-519 (2021). https://doi.org/10.3906/mat-2010-70

33. Baleanu, D., Etemad, S., Rezapour, S.: A hybrid Caputo fractional modeling for thermostat with hybrid boundary value conditions. Bound. Value Probl. 2020, 64 (2020). https://doi.org/10.1186/s13661-020-01361-0

34. Baleanu, D., Etemad, S., Rezapour, S.: On a fractional hybrid integro-differential equation with mixed hybrid integral boundary value conditions by using three operators. Alex. Eng. J. 59(5), 3019-3027 (2020). https://doi.org/10.1016/j.aej.2020.04.053

35. Baleanu, D., Rezapour, S., Saberpour, Z:: On fractional integro-differential inclusions via the extended fractional Caputo-Fabrizio derivation. Bound. Value Probl. 2019, 79 (2019). https://doi.org/10.1186/s13661-019-1194-0

36. Aydogan, S.M., Baleanu, D., Mousalou, A., Rezapour, S.: On high order fractional integro-differential equations including the Caputo-Fabrizio derivative. Bound. Value Probl. 2018, 90 (2018). https://doi.org/10.1186/s13661-018-1008-9

37. Rezapour, S., Samei, M.E.: On the existence of solutions for a multi-singular pointwise defined fractional q-integro-differential equation. Bound. Value Probl. 2020, 38 (2020). https://doi.org/10.1186/s13661-020-01342-3

38. Fawziah, A.S., Ghadle, K.P.: Solving nonlinear Fredholm integro-differential equations via modifications of some numerical methods. Adv. Theory Nonlinear Anal. Appl. 5(2), 260-276 (2021). https://doi.org/10.31197/atnaa.872432

39. Nashine, H.K., Ibrahim, R.W., Can, N.H.: Solution of a fractal energy integral operator without body force using measure of noncompactness. Alex. Eng. J. 59(6), 4101-4106 (2020). https://doi.org/10.1016/j.aej.2020.07.015

40. Raja, M.M., Vijayakumar, V., Udhayakumar, R., Zhou, Y.: A new approach on the approximate controllability of fractional differential evolution equations of order $1<r<2$ in Hilbert spaces. Chaos Solitons Fractals 141, 110310 (2020). https://doi.org/10.1016/j.chaos.2020.110310

41. Raja, M.M., Vijayakumar, V., Udhayakumar, R.: A new approach on approximate controllability of fractional evolution inclusions of order $1<r<2$ with infinite delay. Chaos Solitons Fractals 141, 110343 (2020). https://doi.org/10.1016/j.chaos.2020.110343

42. Rezapour, S., Henriquez, H.R., Vijayakumar, V., Nisar, K.S., Shukla, A.: A note on existence of mild solutions for second-order neutral integro-differential evolution equations with state-dependent delay. Fractal Fract. 5(3), 126 (2021). https://doi.org/10.3390/fractalfract5030126

43. Duraisamy, S.R., Sundararajan, P., Karthikeyan, K.: Controllability problem for fractional impulsive integrodifferential evolution systems of mixed type with the measure of noncompactness. Results Nonlinear Anal. 3(2), 85-99 (2020)

44. Mahmudov, N.I., Denker, A.: On controllability of linear stochastic systems. Int. J. Control 73(2), 144-151 (2000). https://doi.org/10.1080/002071700219849

45. Mahmudov, N.I: Approximate controllability of evolution systems with nonlocal conditions. Int. J. Control 68(3), 536-546 (2008). https://doi.org/10.1016/..na.2006.11.018

46. Mahmudov, N.I., Vijayakumar, V., Murugesu, R.: Approximate controllability of second-order evolution differential inclusions in Hilbert spaces. Mediterr. J. Math. 13, 3433-3454 (2016). https://doi.org/10.1007/s00009-016-0695-7

47. Mahmudov, N.I., Udhayakumar, R., Vijayakumar, V.: On the approximate controllability of second-order evolution hemivariational inequalities. Results Math. 75, 160 (2020). https://doi.org/10.1007/s00025-020-01293-2

48. Naito, K.: Controllability of semilinear control systems dominated by the linear part. SIAM J. Control Optim. 25(3), 715-722 (1987). https://doi.org/10.1137/0325040

49. Nisar, K.S., Vijayakumar, V.: Results concerning to approximate controllability of non-densely defined Sobolev-type Hilfer fractional neutral delay differential system. Math. Methods Appl. Sci. (2021). https://doi.org/10.1002/mma.7647 
50. Sakthivel, R., Ganesh, R., Anthoni, S.M.: Approximate controllability of fractional nonlinear differential inclusions. Appl. Math. Comput. 225(1), 708-717 (2013). https://doi.org/10.1016/j.amc.2013.09.068

51. Shukla, A., Sukavanam, N., Pandey, D.N.: Approximate controllability of fractional semilinear control system of order $\alpha \in(1,2]$ in Hilbert spaces. Nonlinear Stud. 22(1), 131-138 (2015)

52. Shukla, A., Sukavanam, N., Pandey, D.N.: Approximate controllability of semilinear fractional control systems of order $\alpha \in(1,2]$ with infinite delay. Mediterr. J. Math. 13, 2539-2550 (2016). https://doi.org/10.1007/s00009-015-0638-8

53. Shukla, A., Sukavanam, N., Pandey, D.N.: Approximate controllability of fractional semilinear stochastic system of order $\alpha \in(1,2]$. J. Dyn. Control Syst. 23, 679-691 (2017). https://doi.org/10.1007/s10883-016-9350-7

54. Shukla, A., Sukavanam, N., Pandey, D.N., Arora, U.: Approximate controllability of second-order semilinear control system. Circuits Syst. Signal Process. 35, 3339-3354 (2016). https://doi.org/10.1007/s00034-015-0191-5

55. Shukla, A., Patel, R.: Existence and optimal control results for second-order semilinear system in Hilbert spaces. Circuits Syst. Signal Process. 40, 4246-4258 (2021). https://doi.org/10.1007/s00034-021-01680-2

56. Shukla, A., Patel, R.: Controllability results for fractional semilinear delay control systems. J. Appl. Math. Comput. 65(1-2), 861-875 (2021). https://doi.org/10.1007/s12190-020-01418-4

57. Shukla, A., Sukavanam, N., Pandey, D.N.: Approximate controllability of semilinear fractional stochastic control system. Asian-Eur. J. Math. 11(6), 1850088 (2018). https://doi.org/10.1142/S1793557118500882

58. Vijayakumar, V., Henriquez, H.R.: Existence of global solutions for a class of abstract second order nonlocal Cauchy problem with impulsive conditions in Banach spaces. Numer. Funct. Anal. Optim. 39(6), 704-736 (2018). https://doi.org/10.1080/01630563.2017.1414060

59. Vijayakumar, V., Murugesu, R., Poongodi, R., Dhanalakshmi, S.: Controllability of second order impulsive nonlocal Cauchy problem via measure of noncompactness. Mediterr. J. Math. 14(3), 1-23 (2017). https://doi.org/10.1007/s00009-016-0813-6

60. Williams, W.K., Vijayakumar, V., Udhayakumar, R., Nisar, K.S.: A new study on existence and uniqueness of nonlocal fractional delay differential systems of order $1<r<2$ in Banach spaces. Numer. Methods Partial Differ. Equ. 37(2), 949-961 (2021). https://doi.org/10.1002/num.22560

61. Batty, C.J.K., Chill, R., Srivastava, S.: Maximal regularity for second order non-autonomous Cauchy problems. Stud. Math. 189(3), 205-223 (2008). https://doi.org/10.4064/sm189-3-1

62. Henriquez, H.R., Castillo, G.: The Kneser property for the second order functional abstract Cauchy problem. Integral Equ. Oper. Theory 52, 505-525 (2005). https://doi.org/10.1007/s00020-002-1266-9

63. Kisynski, J.: On cosine operator functions and one parameter group of operators. Stud. Math. 44(1), 93-105 (1972)

64. Kozak, M.: A fundamental solution of a second order differential equation in a Banach space. Universitatis Lagellonicae Acta Mathematica 32, 275-289 (1995)

65. Travis, C.C., Webb, G.F.: Compactness, regularity, and uniform continuity properties of strongly continuous cosine families. Houst. J. Math. 3(4), 555-567 (1977)

66. Travis, C.C., Webb, G.F.: Cosine families and abstract nonlinear second order differential equations. Acta Math. Acad. Sci. Hung. 32, 75-96 (1978). https://doi.org/10.1007/BF01902205

67. Kavitha, K., Vijayakumar, V., Udhayakumar, R., Ravichandran, C.: Results on controllability of Hilfer fractional differential equations with infinite delay via measures of noncompactness. Asian J. Control (2020). https://doi.org/10.1002/asjc.2549

68. Kavitha, K., Vijayakumar, V., Udhayakumar, R., Sakthivel, N., Nisar, K.S.: A note on approximate controllability of the Hilfer fractional neutral differential inclusions with infinite delay. Math. Methods Appl. Sci. 44(6), 4428-4447 (2021). https://doi.org/10.1002/mma.7040

69. Henriquez, H.R.: Existence of solutions of non-autonomous second order functional differential equations with infinite delay. Nonlinear Anal., Theory Methods Appl. 74(10), 3333-3352 (2011). https://doi.org/10.1016/j.na.2011.02.010

\section{Submit your manuscript to a SpringerOpen ${ }^{\circ}$ journal and benefit from:}

- Convenient online submission

- Rigorous peer review

- Open access: articles freely available online

- High visibility within the field

Retaining the copyright to your article

Submit your next manuscript at $\gg$ springeropen.com 